\title{
3D Thermal-Aware Floorplanner for Many-Core Single-Chip Systems
}

\author{
David Cuesta \\ Complutense University \\ Madrid, Spain \\ dcuestag@pdi.ucm.es
}

\author{
José L. Risco-Martin \\ Complutense University \\ Madrid, Spain \\ jlrisco@dacya.ucm.es
}

\author{
José L. Ayala \\ Complutense University \\ Madrid, Spain \\ jayala@fdi.ucm.es
}

\author{
David Atienza \\ EPFL \\ Lausanne, Switzerland \\ david.atienza@epf.ch
}

\begin{abstract}
Heat removal and power density distribution delivery have become two major reliability concerns in 3D stacked technology. In this paper, we propose a thermal-driven 3D floorplanner. Our contributions include: (1) a novel multi-objective formulation to consider the thermal and performance constraints in the optimization approach; (2) an efficient Mixed Integer Linear Programming (MILP) representation of the floorplanning model; and (3) a smooth integration of the MILP model with an accurate thermal modelling of the architecture. The experimental work is conducted for two realistic many-core single-chip architectures: an homogeneous system resembling Intel's SCC, and an improved heterogeneous setup. The results show promising improvements of the mean, peak temperature and the thermal gradient, with a reduced overhead in the wire length of the system.
\end{abstract}

\section{INTRODUCTION}

Current computer architectures have reached a performance barrier. Patterson has formulated the problem as follows: The power wall + the memory wall + the ILP wall $=$ a brick wall for serial performance. This can be understood like the limit in materials and architectures to provide increasing throughput. Therefore, computer architects have been forced to turn to parallel architectures to continue to make progress. Parallelism can be exploited by using the additional transistors (forecasted by Moores law) to add more independent CPUs, data-parallel execution units, additional registers sets for hardware threads, bigger caches, and more independent memory controllers to increase memory bandwidth.

The emergence of heterogeneous many core architectures presents a unique opportunity for delivering order of magnitude performance increases to high performance applications by matching certain classes of algorithms to specif cally tailored architectures. Specif c HPC applications like N-Body Simulations, Molecular Dynamics, and Terrain Rendering [1] can experience order of magnitude or greater speedups when paired with architectures that are specif cally tailored to their needs. Similar examples from the HPC community include the Los Alamos National Labs Roadrunner system.

This trend on executing the target applications in many parallel cores is not only one of the characteristics of the current datacenters, but also multi-processor systems-on-chip (MPSoCs) have reached the category of many-core systems. Intel Labs has created an experimental Single-chip Cloud Computer, (SCC) a research microprocessor containing the most Intel Architecture cores ever integrated on a silicon CPU chip 48 cores. [2] It incorporates technologies intended to scale multicore processors to 100 cores and beyond, such as an onchip network, advanced power management technologies and support for message-passing.

The exponentially increasing power densities that have been reached in current technologies, the values of leakage currents, the cooling costs and the recent reliability constraints in microprocessor-based systems have motivated the cooling down of the chip temperature to be one of the main concerns in system design. The operating temperature has a signif cant impact on microprocessor design. At higher temperatures, transistors work slower due to the degradation of the carrier mobility. The resistivity of the metal interconnects also increases, causing longer delays and performance degradation. Reliability is also strongly related to temperature, and increasing the temperature exponentially decreases the chip lifetime. In fact, time to failure is a function of $e^{-E a / k T}$, where $E a$ is the activation energy of the failure mechanism and accelerated by temperature increase, $k$ is the Boltzmann's constant, and T is the absolute temperature. When the operating temperature exceeds a threshold, the effect on reliability can be permanent and seriously impact lifetime. Each 10 degrees rise reduces the life of the component by a $50 \%$. Hence, lower operating temperatures of components maximize reliability.

In order to maintain the chip temperature under a certain limit, the power density of the hardware modules can be decremented by increasing the chip area. However, this is not admissible in terms of cost, and the problem of meeting all the geometric constraints should be solved.

Orthogonal to the power density of the functional blocks, another important factor that affects the temperature distribution of the chip is the lateral spreading of heat in silicon. This depends on the placement of the units and their proximity to the chip border and other units that behave as thermal sinks or thermal sources. Thermal-aware f oorplanning algorithms are able to even out the temperature of the hardware modules through spreading of the heat dissipation. This aspect of foorplanning is particularly attractive in comparison with static external cooling, that reduces the temperature of the chip surface by a constant factor (it does not reduce the temperature gradient across the chip).

Three-dimensional (3D) multi-processor chips have been proposed as an effective mechanism to signif cantly improve 
system performance by reducing interconnect delays and increasing the density of the integrated logic, turning the "manycore single-chip" into a reality. It also allows the integration of multiple and disparate technologies, such as radio frequency and mixed signal components.

A major concern in the adoption of $3 \mathrm{D}$ architectures is the increased power densities that can result from placing one computational block over another in the multi-layered 3D stack. Also, the thermal conductivity of the dielectric layers inserted between device layers for insulation is very low compared to silicon and metal. Since power densities are already a major concern in $2 \mathrm{D}$ architectures, the move to 3D architectures will accentuate the thermal problem. Consequently, it is mandatory to devise eff cient 3D foorplanning mechanisms that optimize the thermal prof le of these complex 3D multi-processor architectures.

This work continues the initiated in [3] that proposes a set of design rules for the generation of thermal-aware foorplans for the 3D Niagara architecture. This previous work obtained improvements of the thermal metrics with respect to the baseline architecture and compared to traditional thermal-aware foorplanner. The work presented in this paper outperforms this results with a MILP formulation and an eff cient solver that manages multiple objectives in the minimization problem, as well as considering a many-core heterogeneous single-chip for experimental purposes.

This paper specif cally makes the following contributions:

1) it provides a novel multi-objective formulation of the foorplanning problem in 3D multi-processor architectures with thermal constraints.

2) it performs an eff cient resolution of the optimization problem by the use of a Mixed Integer Linear Programming (MILP) framework.

3 ) it shows good response in terms of the main thermal metrics (mean temperature, peak temperature and thermal gradient) for a many-core homogeneous and heterogeneous single-chip architecture that resembles the Intel's SCC.

\section{RELATED WORK}

The impact of the foorplanning on the thermal distribution of real microprocessor-based systems is analyzed in [4], where the placement of components for Alpha and Pentium Pro is evaluated. Some initial works on thermal aware foorplanning [5] propose a combinatorial optimization problem to model our problem. However, the simplif cation of the considered f oorplan and the lack of a real experimental framework motivated the further research on the area. Thermal placement for standard cell ASICs is a well researched area in the VLSI CAD community, where we can f nd works as [6].

In the area of f oorplanning for microprocessor-based systems, some authors consider the problem at the microarchitectural level [7], where it is shown that signif cant peak temperature reduction can be achieved by managing lateral heat spreading through f oorplanning. Other works [8] use genetic algorithms to demonstrate how to decrease the peak temperature while generating foorplans with area comparable to that achieved by traditional techniques. [9] uses a simulated annealing algorithm and an interconnect model to achieve thermal optimization. These works have a major restriction since they do not consider multiple objective factors in the optimization problem, as opposed to our work. Our proposed foorplanner optimizes jointly both thermal metrics (mean temperature, peak temperature and gradient) with a strong impact on the reliability of the system, and the performance of the system (through the minimization of the wire length delay). Moreover, the thermal models used in these studies do not ref ect the complex diffusion processes that exist in current technologies. More recent works [10] have tackled the problem of thermalaware foorplanning with geometric programming but, in this case, the area of the chip is not considered constant.

Thermal-aware foorplanning for 3D stacked systems has also been investigated. Cong et al. [11] proposed a thermaldriven foorplanning algorithm for 3D ICs, which is a natural extension of his previous work on 2D. In [12], Healy et al. implemented a multi-objective foorplanning algorithm for $2 \mathrm{D}$ and $3 \mathrm{D}$ ICs, combining linear programming and simulated annealing.

Our work presents more similarities with the reference [13] by Hung, where they propose a thermal-aware foorplanner for $3 \mathrm{D}$ architectures. However, this preliminary work does not consider the multi-objective approach proposed in our work, and does not consider the minimization of those thermal variables with a strong impact on the reliability of the system.

Thus, an eff cient model of the optimization problem and an effective solver are required to achieve good trade-offs between thermal optimization and performance constrains. In the case of 3D IC design, incremental optimization is a promising way to handle multi-objective optimization with complicated constraints and facilitate the design reuse technology. Several works concerned with incremental foorplanning for 2D IC design [14], [15] have been proposed, but none has taken thermal-aware 3D IC design into consideration. [16] has recently proposed an incremental MILP algorithm. However the design process could take several iterations, whereas our methodology perform the thermal-aware and total wire length optimization in two steps.

In this paper, we propose a novel algorithm to optimize the 3D layout in order to eliminate the hotspots, reduce the peak temperature and decrease the reliability risks. Given a $3 \mathrm{D}$ packing and a chip area, we formulate the thermal-aware and total wire length optimization into two MILP problems. The former is def ned in terms of power density, moving hottest blocks until they are as far away as possible from each other. Then, with hottest blocks fxed in space, we perform another optimization trying to move the remaining blocks to reduce total wire length. Experiments results show that we can reduce the maximum on-chip temperature in 54 degrees on average for two realistic homogeneous and heterogeneous many-core single-chip architectures, outperforming previous thermal-aware foorplan designs. 


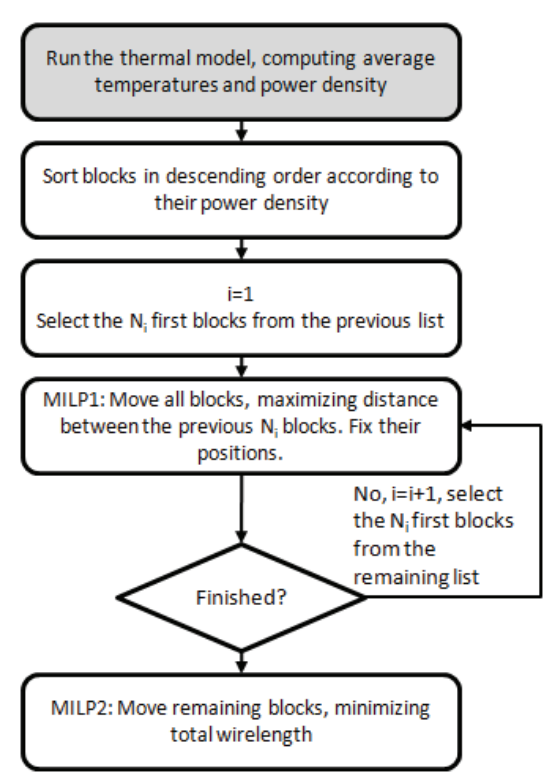

Fig. 1. Iterative fow of our approach

\section{DESIGN FLOW}

In order to reduce maximal on-chip temperature as much as possible, we propose a novel thermal-aware incremental optimization fow. To this end, we have developed three algorithms. The frst one performs an accurate analysis of the thermal behavior in the 3D IC. The second moves all the blocks until the hottest ones are as far away as possible from each other. The last one, having f xed the hottest, tries to move the remaining blocks while total wire length is minimized.

Fig. 1 shows the design fow of our thermal-aware 3D microarchitectural foorplanner. Such fow can be divided in two phases. The former is the thermal analysis of an initial conf guration of the 3D IC (gray shaded block in Fig. 1). Since we are studying the Niagara system, such initial conf guration is available in [17]. If not, we can obtain it by running the second MILP algorithm proposed in this work (last block in Fig. 1). The second phase is the optimization loop (rest of the diagram). Next, we describe these two phases in detail.

\section{A. Thermal analysis}

As Fig. 1 shows, we frst perform a thermal analysis. To this end, we have developed an accurate thermal model, which is brief y described in the following.

3D integration consists on placing different active layers using silicon dioxide and joining them with a glue material. If inter layer communication is required, Through Silicon Vias (TSVs) allow it. Some of the goals on the design of 3D stacks are to achieve The 3D stack is built over an adiabatic PCB surface and then, traditional technological dies composed by silicon dioxide and silicon, are placed one over the others.

The heat fow through the $3 \mathrm{D}$ stack is diffusive, hence, it can be characterized with a $3 \mathrm{D}$ RC thermal model as the one presented in [18].

The thermal modeling of the stack is performed after splitting the chip into small cubic unitary cells. These cells are modeled with six thermal resistances and one thermal capacitance as

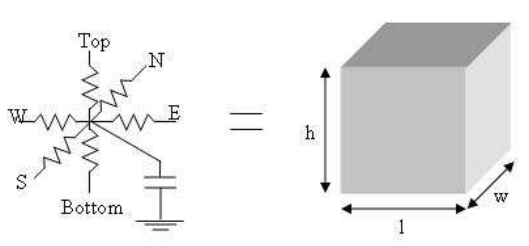

Fig. 2. Equivalent RC circuit of a single cell

can be seen in Figure 2. Four of these resistances connect each cell to its lateral neighbors (those on the same layer), while the two remaining resistances connect the cell with the upper and bottom cell, respectively. The capacitance represents the heat storage inside the cell. The values of the conductances and the capacitance are calculated using these expressions:

$$
\begin{gathered}
G_{\text {top } / \text { bottom }}=k_{t h}(l \cdot w) /(h / 2) \\
G_{\text {north } / \text { south }}=k_{t h}(l \cdot w) /(h / 2) \\
G_{\text {east } / \text { west }}=k_{t h}(l \cdot w) /(h / 2) \\
C_{t o p}=s c_{t h}(l \cdot w \cdot h)
\end{gathered}
$$

where north, south, east and west indicate the direction in which heat is diffused; $k_{t h}$ and $s c_{t h}$ are the thermal conductivity and specif c heat capacity per volume unit of the material, respectively.

The model also considers the heat diffusion to the surrounding environment. The existence of TSVs is considered in the model, also as a resistance element. The most important thermal properties of the material used in the model are listed in Table I.

TABLE I

THERMAL PROPERTIES OF MATERIALS

\begin{tabular}{cc}
\hline Silicon linear thermal conductivity & $295 \mathrm{~W} /(\mathrm{mK})$ \\
Silicon quadratic thermal conductivity & $-0.491 \mathrm{~W} /\left(\mathrm{m} K^{2}\right)$ \\
Silicon dioxide thermal conductivity & $1.38 \mathrm{~W} /(\mu \mathrm{mK})$ \\
Silicon specif c heat & $1.628 \times 10^{6} \mathrm{~J} / \mathrm{m}^{3} \mathrm{~K}$ \\
Silicon dioxide specif c heat & $4.180 \times 10^{6} \mathrm{~J} / \mathrm{m}^{3} \mathrm{~K}$
\end{tabular}

Once the resistance and capacitance values for every unitary cell are calculated, a set of equations that describe the RC grid is created. After that, an iterative method (Forward Euler) is used to solve it.

The active elements in the $3 \mathrm{D}$ stack can be considered as heat sources or heat sinks. Processors are considered strong heat sources, they dissipate power in the die, and this heat is then spread throughout the chip. On the other hand, memories have a lower power activity and they can be considered almost as heat sinks. This is an important consideration to be taken into account since the foorplanner will try to place both heat sinks and heat sources as close as possible (provided the routing and performance constraints) to balance the thermal profle.

Once the previous model has been applied to the 3D IC, we obtain mean and peak temperatures, as well as the thermal gradient and power density, which are used later in the optimization phase. 


\section{B. Optimization phase}

The proposed approach tries to minimize two conficting objectives: maximum temperature $\left(J_{1}\right)$ and total wire length $\left(J_{2}\right)$. Is this article, instead of minimizing a weighted sum of both objectives like in previous approaches (see for example [16]), we perform a multi-objective optimization. Thus, the f rst question is: Which search algorithms should be used? The f oorplanning optimization problem is NP-hard. As a result, a solver could not $\mathrm{f}$ nd the global optimum in a f nite time. There are several approaches to perform such optimization eff ciently. Examples are the use of meta-heuristics (like simulated annealing, genetic algorithms, particle swarm optimizers, etc.), as well as classic methodologies based on MILP. We used MILP because of two main reasons: (1) MILP solvers immediately check if a design scenario is or not feasible, and (2) if the problem is correctly formulated, MILP quickly offers feasible solutions.

To perform a multi-objective optimization in MILP, we proceed as follows. In general terms, all the problem objectives, except one, are introduced into the set of constraints to arbitrarily give a value in the right side of each new constraint (one per objective). Let us suppose that $V_{2}$, corresponds to the value of $J_{2}$ (total wire length) when $J_{1}$ (maximum temperature) is minimized. If the constraint $J_{2}=V_{2}$ is added and the problem is solved again, it would once again obtain the same solution for $J_{1}$. But if the constraint $J_{2} \leq V_{2}-\epsilon$ is added where $\epsilon$ is a relatively small positive value, and the problem is solved, it is possible that the new solution of $J_{1}$ is superior or equal, but obviously never inferior, since when adding a new constraint, the number of feasible solutions is reduced. Therefore, as the value of $J_{2}$ is decreased and new problem instances are solved, new solutions for $J_{1}$ are generated. The process stops when the right side of the constraint, $V_{2}-\epsilon$, reaches the optimal value of $J_{2}$. The problem resides in f nding the value of $\epsilon$ adapted to being able to generate the maximum number of eff cient points in the space of objectives. In our case, since we are trying to outperform an initial conf guration (i.e., the input to the thermal model) obtaining better solutions for both maximum temperature and wire length, the value of $\epsilon$ can be easily obtained without running the MILP algorithms several times.

To develop a MILP model, we must perform several linear approximations. The frst one is related to the thermal model, which includes non-linear and differential equations. The temperature of an element in an integrated circuit depends on the power density of the element and the proximity of its neighbors. The f rst factor of the equality refers to the increase on the thermal energy due to the activity of the element, while the second factor is related with the diffusion process of heat [19]. Thus, we use the power density of each block as an approximation of its temperature in the steady state. The second approximation is the distance between elements, which is approximated as the Manhattan distance.

Continuing with the optimization phase (second block of Fig. 1) and after analyze the temperature contribution from different blocks, we frst sort the blocks in descending order according to their power density. Next, we select the frst $N_{i}, i=1$ blocks from the previous list, which will be the hottest ones. The selection of these $N_{i}$ blocks is user-def ned. In our experiments we selected all the cores, because their positions decide the f nal temperature distribution along the 3D IC. Next, the frst search algorithm (called MILP1 in Fig. 1) allocates all the blocks, trying to maximize the Manhattan distance $\left(d_{m}\right)$ between the aforementioned $N_{i}$ modules:

$$
\operatorname{maximize} J_{1}=\sum_{i, j \in N_{i}, i<j} d_{m}\left(b_{i}, b_{j}\right)
$$

Next, the optimization can be repeated several times allocating the following set of $N_{i}, i=2$ blocks of the remaining sorted list (having the previous $N_{1}$ blocks fxed in the f nal design). This procedure can be repeated until the sorted list is empty. Finally, we move the remaining blocks (considered those with lowest power density) using a second algorithm (called MILP2 in Fig. 1). In this phase, we do not maximize distance. Instead, we minimize total wire length, approximated as the Manhattan distance between connected blocks $(C)$.

$$
\operatorname{minimize} J_{2}=\sum_{i, j \in C, i<j} d_{m}\left(b_{i}, b_{j}\right)
$$

In the following, we will def ne the experimental set-up, showing the foorplans that will be thermally analyzed and compared with the results obtained by our f oorplanner.

\section{EXPERIMENTAL SET-UP}

The 3D multiprocessor systems studied in our experimental work are based on the Intel's SCC architecture but their processing units are SPARC cores, like those in the Niagara architecture, fabricated in $90 \mathrm{~nm}$ technology (these cores are much more powerful than the Power cores found in SCC and can also easily exhibit higher thermal issues). This architecture has been modif ed to include an increased number of cores, placed in several layers of the 3D stack. Since our f oorplanner can place a variable number of cores in every layer, the area and power consumption of the crossbar is scaled accordingly to the number of cores found in every layer and their required bandwidth. The inter-layer communication is resolved with a set of TSVs that route the communication signals.

The foorplanner will place the functional units that compose the $3 \mathrm{D}$ multi-processor architecture targeting both temperature and wire length optimization. The thermally-optimized foorplans proposed by the foorplanner will be compared with the original conf guration presented in Figure 3. The 64 cores (C) are disposed in 5 layers, where also the L2 memories (L2), the shared memories (L2B) and the crossbar (Cross) can be seen.

The experimental work will analyze the thermal optimization achieved by the foorplanner in two different scenarios. The frst scenario resembles the SCC architecture with a system where 64 SPARC cores are integrated in the 3D stack.

The second scenario models an heterogeneous system where the 64 cores are composed of 48 SPARC and 16 Power6. The 
Power cores are placed instead of cores C2,7 and 12 in the case of inner layers, and C2,7,11 and 12 for the top layer. This setup will show the optimized thermal prof le that can be expected when multiple core architectures are considered, as well as the extra optimization opportunities that the foorplanner will $\mathrm{f}$ nd.

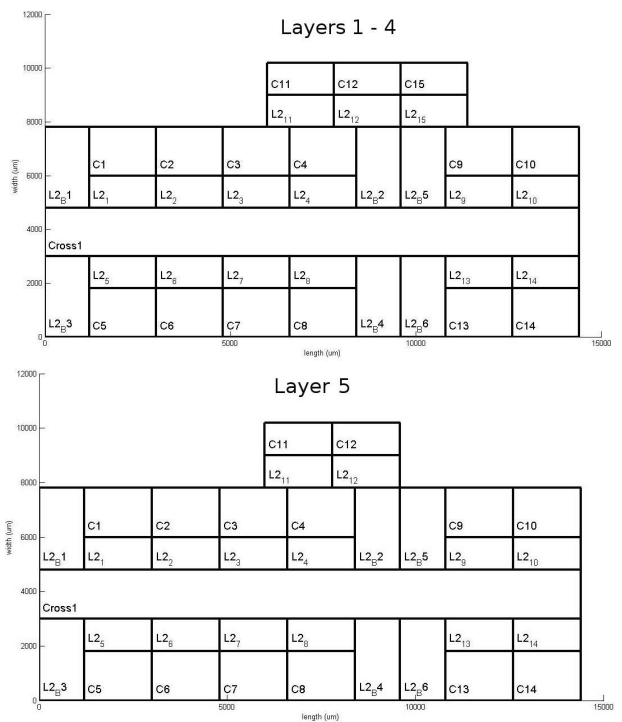

Fig. 3. Original foorplan.

\section{RESUlts}

This section frstly presents the thermal profle of the two scenarios described in Section IV estimated by the thermal model.

The metrics considered for the analysis of the experimental results are the wire length, mean and maximum temperature of the layer, and the maximum thermal gradient. These metrics are usually found in all the thermal-related analysis.

Then, these results will be compared with the thermal prof les exhibited by the outputs of the foorplanner.

The worst case of power consumption in the Niagara2 $(84 \mathrm{~W}$ at $1.1 \mathrm{~V}$ and $1.4 \mathrm{GHz}$ [17]) is considered to extract the power densities of every SPARC unit. Also, the area of the layers has been scaled according to the number of cores and the number of layers has bem increased. The power of the Power6 cores is $2.6 \mathrm{~W}$, as found in [20].

\section{A. Scenario 1}

Figure 4 shows the thermal maps for the simulation of the homogeneous system. The previously def ned thermal metrics, the mean temperature, thermal gradient and maximum temperature for every layer of the conf guration, have been calculated. The comparison with the baseline homogeneous system shows that the foorplanner is capable of optimizing the maximum temperature in 55 degrees, the mean temperature in 24 and the thermal gradient is decreased in 70 .

This can be explained because our foorplanner separates heat sources (cores) as much as possible, trying to place them at the border of the chip, helping on the cooling down of the cores. The foorplanner also takes into account vertical heat
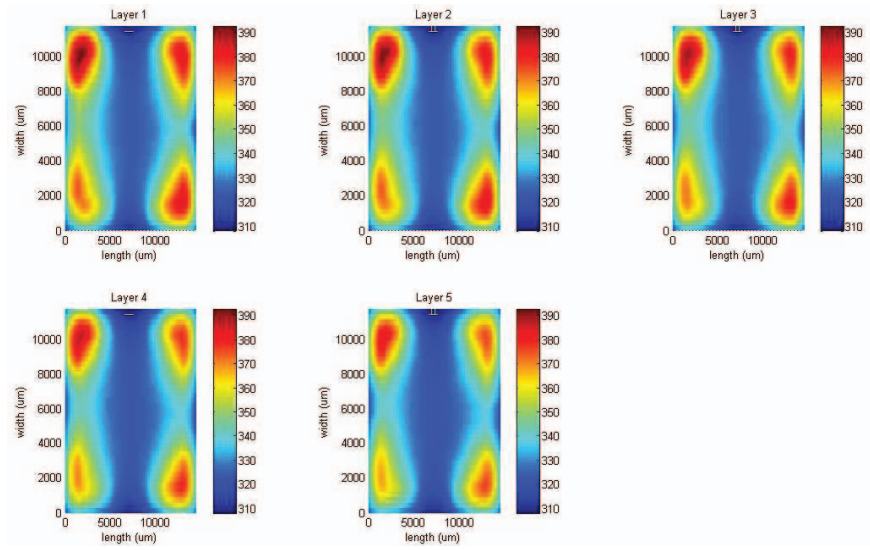

Fig. 4. Thermal maps of the optimized homogeneous system.
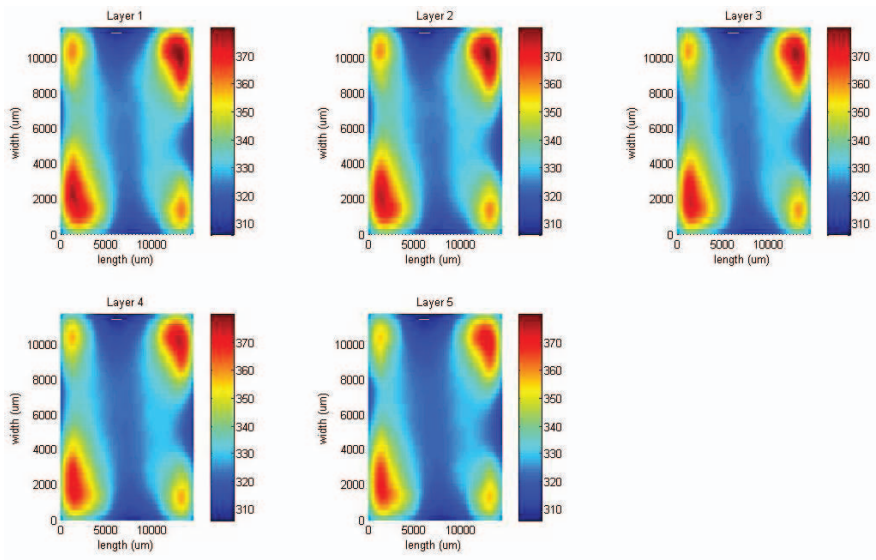

Fig. 5. Thermal maps of the optimized heterogeneous system.

spread, and each layer will have a different layout, avoiding placing heat sources one over the other.

TABLE II

THERMAL DEVIATION (K)

\begin{tabular}{|c|c|c|c|c|c|}
\hline Scenario & Layer 1 & Layer 2 & Layer 3 & Layer4 & Layer 5 \\
\hline S.1Original & 33.74 & 33.34 & 32.55 & 31.37 & 29.79 \\
S.1Floorplanner & 17.75 & 17.46 & 16.90 & 16.51 & 15.89 \\
\hline \hline S.2Original & 28.65 & 28.31 & 27.62 & 26.60 & 25.21 \\
S.2Floorplanner & 14.61 & 14.32 & 13.89 & 13.67 & 13.31 \\
\hline
\end{tabular}

Also, as shown in Table II, the reduced deviation of temperatures across the layers (or spatial thermal gradients) determines a more homogeneous thermal distribution, which is translated into a reduced reliability risk and diminished leakage currents.

\section{B. Scenario 2}

Figure 5 shows the thermal maps for the simulation of the heterogeneous system. Similarly to the previous setup, the mean temperature, thermal gradient and maximum temperature for every layer of the conf guration have been calculated. The comparison with the baseline heterogeneous system shows that the foorplanner is capable of optimizing the maximum temperature in 52 degrees, the mean temperature in 23 and the 
thermal gradient is decreased in 78. Also, the heterogeneous architecture outperforms the results of the homogeneous system in 13 degrees for the maximum temperature, 7 for the mean temperature and 10 for the thermal gradient.

In this case, our foorplanner will try again to maximize the distance between the heat sources. However, the Power cores will not be considered as hotspot by the optimizer since their temperature is much lower than the SPARC's temperature. Therefore, the foorplanner will place the warm Power cores between actual hotspots, achieving a better thermal profle.

Also, as shown in Table II, the reduced deviation of temperatures across the layers determines a more homogeneous thermal distribution, which is translated into a reduced reliability risk and diminished leakage currents.

Table III shows the wirelength associated with the 3D system. As can be seen, the overhead incurred by the foorplanner has been a $24 \%$ when compared to the original distribution for Scenario 1 and $31 \%$ for Scenario 2. This overhead in the wiring is not directly translated into an increase of the communication delay because core-to-core communication is regulated by the crossbar. As the crossbar is the module that limits the bandwidth and speed of the link, this overhead is seen minimized. On the other hand, the big savings reached in the mean temperature (35\% and $41 \%$ for the Scenario 1 and 2 , respectively) justify the overhead in wiring.

TABLE III

WIRE LENGTH

\begin{tabular}{|c|c|c|c|}
\hline $\begin{array}{c}\text { Number } \\
\text { of Layers }\end{array}$ & Floorplan & Wire Length (um) & $\begin{array}{c}\text { Mean } \\
\text { Temperature (K) }\end{array}$ \\
\hline & Original & 3742 & 366 \\
Scenario1 & Floorplanner & 4644 & 343 \\
\hline \hline & Original & 3742 & 356 \\
Scenario2 & Floorplanner & 4918 & 333 \\
\hline
\end{tabular}

\section{Comparison of results}

The results obtained by our algorithm have been compared with those extracted by traditional placement algorithms. For these comparison purposes, a Genetic Algorithm (GA) with reverse polish notation that performs the placement of units has been selected, as seen in previously published works. Table IV shows the results obtained by our algorithm and the GA for two chip conf gurations with 16 and 64 cores, respectively.

TABLE IV

COMPARISON OF RESULTS

\begin{tabular}{|c|c|c|c|c|}
\hline \# cores & Mean T MILP & Wire MILP & Mean T GA & Wire GA \\
\hline 16 & 325 & 595 & 329 & 738 \\
\hline 64 & 333 & 4644 & NA & NA \\
\hline
\end{tabular}

As can be seen, our algorithm not only achieves better results in terms of mean temperature and wiring length, but also it is able to target more complex systems. The GA was not able to converge to a solution in the case of a chip with 64 cores after 6 days of running.

\section{CONCLUSIONS}

This paper has proposed a novel MILP formulation to cope with the problem of thermal-aware f oorplanning in 3D manycore sigle-chips. Also, the eff cient solver that provides the optimization of the foorplan, interfaces with an accurate thermal model, providing promising results in the minimization of the main thermal- and reliability-related metrics (peak and mean temperature, thermal gradients) with low performance overhead. The experimental results have been obtained for two realistic many-core single-chip architectures: an homogeneous system resembling Intel's SCC, and an improved heterogeneous setup. These results outperform previous results obtained by traditional thermal-aware foorplanners.

\section{ACKNOWLEDGMENT}

This work is partially supported by the Spanish Government Research Grant TIN2008-00508. Also, it is partially supported by the Nano-Tera RTD project CMOSAIC (ref.123618), fnanced by the Swiss Confederation and scientif cally evaluated by SNSF, and the PRO3D EU FP7-ICT-248776 project.

\section{REFERENCES}

[1] V. T. B. Minor and G. Fossum, “Terrain Rendering Engine (TRE)," IBM, Tech. Rep., 2005.

[2] Intel Labs, SCC External Architecture Specification (EAS), May 2010.

[3] D. Cuesta, J. L. Ayala, J. Hidalgo, M. Poncino, A. Acquaviva, and E. Macii, "Thermal-aware foorplanning exploration for 3D multi-core architectures," in GLSVLSI, 2010, pp. 99-102.

[4] I. K. Y. Han and C. Moritz, "Temperature aware foorplanning," in Workshop on Temperature-Aware Computer Systems, 2005.

[5] C. C. N. Chu and D. F. Wong, "A matrix synthesis approach to thermal placement," in International Symposium on Physical Design, 1997.

[6] G. Chen and S. Sapanetkar, "Partition-driven standard cell thermal placement," in International Symposium on Physical Design, p. 2003.

[7] K. Sankaranarayanan, S. Velusamy, M. R. Stan, and K. Skadron, "A case for thermal-aware f oorplanning at the microarchitectural level," Journal of Instruction Level Parallelism, vol. 7, pp. 8-16, 2005.

[8] W.-L. Hung, Y. Xie, N. Vijaykrishnan, C. Addo-Quaye, T. Theocharides, and M. J. Irwin, "Thermal-aware foorplanning using genetic algorithms," in International Symposium on Quality Electronic Design, 2005.

[9] Y. Han and I. Koren, "Simulated annealing based temperature aware f oorplanning," Journal of Low Power Electronics, vol. 3, no. 2, pp. 1-15, 2007.

[10] Y.-C. Chen and Y. Li, "Thermal-aware foorplanning via geometric programming," Mathematical and Computer Modelling, vol. 51, pp. 927-934, 2010.

[11] J. Cong, J. Wei, and Y. Zhang, "A thermal-driven f oorplanning algorithm for $3 \mathrm{~d}$ ics," in ICCAD, 2004.

[12] M. Healy et al., "Multiobjective microarchitectural foorplanning for 2-d and 3-d ics," Trans. on CAD, vol. 26, pp. 38-52, 2007.

[13] W.-L. Hung et al., "Interconnect and thermal-aware foorplanning for 3d microprocessors," in ISOED, 2006, pp. 98-104.

[14] J. Cong and M. Sarrafzadeh, "Incremental physical design," in ISPD, 2000.

[15] P. B. J. Creshaw, M. Sarrafzadeh and P. Prabhakaran, "An incremental f oorplanner," in GLSVLSI, 1999.

[16] Y. M. X. Li and X. Hong, "A novel thermal optimization fow using incremental foorplanning for $3 \mathrm{~d}$ ics," in ASPDAC, 2009, pp. 347-352.

[17] http://www.opensparc.net/pubs/preszo/07/n2isscc.pdf.

[18] J. L. Ayala et al., "Through Silicon Via-Based Grid for Thermal Control in 3D Chips," in Nano-Net, ser. Lecture Notes in Computer Science, vol. 1, no. 1. Springer, 2009, pp. 1-7.

[19] L. B. G. Paci, F. Poletti and P. Marchal, "Exploring temperature-aware design in low-power mpsocs," IJES International journal of embedded systems, vol. 3, no. 1, pp. 43-51, 2007.

[20] "IBM Systems Energy Estimator," http://www.ibm.com/systems/ support/tools/estimator/energy. 